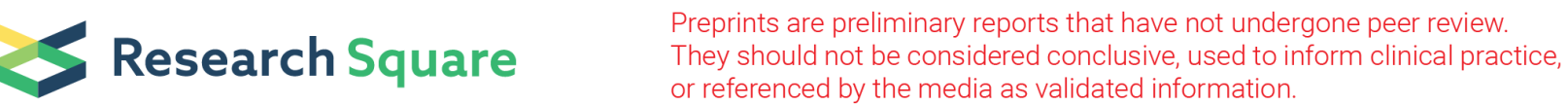

\section{Long Noncoding RNA GAS6-AS1 Inhibits Progression and Glucose Metabolism Reprogramming in Lung Adenocarcinoma Via Repressing E2F1-Mediated Transcription of GLUT1}

Jing Luo

Jiangsu Cancer Hospital

Huishan Wang

Shanghai Songjiang District Central Hospital

\section{Li Wang}

Nanjing medical university

\section{Gaoming Wang}

Nanjing Jinling Hospital: East Region Military Command General Hospital

Yu Yao

Nanjing Second Hospital

Kai Xie

Nanjing Jinling Hospital: East Region Military Command General Hospital

Xiaokun Li

Nanjing Jinling Hospital: East Region Military Command General Hospital

Lin Xu ( $\nabla$ xulin83@njmu.edu.cn )

Jiangsu Cancer Hospital

Yi Shen

Nanjing Jinling Hospital: East Region Military Command General Hospital

Binhui Ren

Jiangsu Cancer Hospital

Research

Keywords: GAS6-AS1, lung adenocarcinoma, glucose metabolism, E2F1, GLUT1

Posted Date: September 23rd, 2020

DOI: https://doi.org/10.21203/rs.3.rs-79477/v1

License: (c) (1) This work is licensed under a Creative Commons Attribution 4.0 International License.

Read Full License 
Version of Record: A version of this preprint was published at Molecular Therapy - Nucleic Acids on September 1st, 2021. See the published version at https://doi.org/10.1016/j.omtn.2021.04.022. 


\section{Abstract}

\section{Background}

Glucose metabolism reprogramming is one of the hallmarks of cancer cells. While functional and regulatory mechanism of long noncoding RNA (IncRNA) in the contribution of glucose metabolism in lung adenocarcinoma (LUAD) remains incompletely understood. The aim of this study was to uncover the roles for GAS6-AS1 in the regulation of progression and glucose metabolism in LUAD.

\section{Methods}

The tumor-suppressive function of GAS6-AS1 was determined by experiments in vitro and nude mice xenograft models. The role of GAS6-AS1 in regulating cancer glucose metabolism was proved by detecting glucose uptake, lactate production, pyruvate production and extracellular acidification rate (ECAR). RNA pull-down assay, RNA immunoprecipitation (RIP) assay, luciferase reporter assay and Chromatin Immunoprecipitation (ChIP) assay were used to identify the underlying molecular mechanisms of GAS6-AS1. And the expression level of GAS6-AS1 in LUAD tissues and cells was measured by quantitative real-time PCR.

\section{Results}

Overexpression of GAS6-AS1 suppressed tumor progression of LUAD both in vitro and in vivo. Metabolicrelated assays revealed that GAS6-AS1 inhibited glucose metabolism reprogramming. Mechanically, GAS6-AS1 was found to repress the expression of glucose transporter GLUT1, a key regulator of glucose metabolism. Ectopic expression of GLUT1 restored the inhibition effect of GAS6-AS1 on cancer progression and glucose metabolism reprogramming. Further investigation identified that GAS6-AS1 directly interacted with transcription factor E2F1 and suppressed E2F1-mediated transcription of GLUT1. And GAS6-AS1 was downregulated in LUAD tissues and correlated with clinicopathological characteristics and survival of patients.

\section{Conclusions}

Taken together, our results identified GAS6-AS1 as a novel tumor suppressor in LUAD and unraveled its underlying molecular mechanism in reprogramming glucose metabolism. GAS6-AS1 potentially served as a prognostic marker and therapeutic target in LUAD.

\section{Introduction}

The rapid proliferation and growth of solid tumors lead to a shortage of energy supply and an oxygen deprivation. Glucose is the major energy source to support tumor growth and can also provide carbon source for biosynthetic reactions[1]. Cancer cells exhibit dramatic aberration of glucose metabolism, displaying activation of glycolysis despite the presence of oxygen, also known as the "Warburg effect"[2]. In glycolysis, cancer cells produce adenosine triphosphate (ATP) through pyruvate transformation to 
lactic acid in the cytosol, being characterized by more uptake of glucose, more production of lactate and pyruvate, and an extra-cellular acidic $\mathrm{pH}[3]$. Recently, glucose metabolism reprogramming has been recognized as a hallmark of cancer and enhanced glycolytic effect is proved to support the survival and growth of cancer cells[4]. Given the crucial role played by glucose metabolism in cancer biology, it is necessary to identify key molecules or pathways that reprogram this process.

Long noncoding RNAs (IncRNAs) represent a large class of transcripts greater than 200 nucleotides with no protein coding capability[5]. Current studies have uncovered the vital roles of IncRNAs in transcription, genomic imprinting, messenger RNA degradation, translation, protein kinetics or function as RNA decoys or scaffolds[6]. And increasing evidence demonstrates that IncRNAs function in the pathogenesis of cancers with diverse mechanisms[7, 8]. However, the potential role and mechanism of IncRNAs in the regulation of cancer metabolism remain largely unknown.

Lung cancer is the most common malignancy and the leading cause of cancer-related death worldwide[9]. Two main groups of lung cancer are small-cell lung cancer (SCLC, 15-20\% cases) and nonsmall cell lung cancer (NSCLC, 80-85\% cases). And lung adenocarcinoma (LUAD) represents the most common histological subtype of NSCLC, accounting for around 40\% [10]. Multiple researches have confirmed the enhancement of glycolysis in LUAD [11-13] but the specific mechanisms of glucose metabolism reprogramming in LUAD need to be further teased out. The aim of this study is to probe metabolism-related IncRNA in LUAD and to study its functional mechanism. Herein we identified GAS6AS1 as a metabolism-regulating IncRNA which was dysregulated in LUAD tissues and meanwhile in glucose-starved LUAD cells. Mechanistically, GAS6-AS1 exerted its effect on progression and glucose metabolism via repressing E2F1-mediated transcription of GLUT1. Glucose transporter 1 (GLUT1), a pivotal rate-limiting element in the transport of glucose in malignancy cells, has been extensively documented to regulate glucose metabolism[14]. Our results indicate that the GAS6-AS1/E2F1/GLUT1 axis may be a promising metabolism target for antitumor therapy.

\section{Materials And Methods Bioinformatics}

GEO microarray dataset GSE56843 (https://www.ncbi.nlm.nih.gov/geo/query/acc.cgi?acc=GSE56843) and GEPIA database (http://gepia2.cancer-pku.cn/\#index)[15] were used to screen metabolism-related IncRNAs in LUAD. Gene clusters (TableS1) highly co-expressed with GAS6-AS1 (Pearson Correlation Coefficient $\geq 0.35$ ) were submitted to DAVID Bioinformatics Resources 6.8 (http://david.abcc.ncifcrf.gov/)[16] for Gene_Ontology enrichment analysis. LncRNA Modulator Atlas in Pan-cancer (LncMAP) [17] were browsed to seek for transcription factors potentially interacting with GAS6-AS1. The secondary structure of GAS6-AS1 was predicted in AnnoLnc (http://annolnc.gao-lab.org/) [18] and catRAPID (http://s.tartaglialab.com/page/catrapid_group)[19] was explored to estimate the binding propensity of GAS6-AS1 with E2F1. The Jaspar database (http://jaspardev.genereg.net/)[20] was used to evaluate the transcription factor binding profiles with gene promoters. And CCLE database 
(https://portals.broadinstitute.org/ccle/about) were searched to verify the expression of GAS6-AS1 in different cancer cell lines.

\section{Patient specimens}

80 paired LUAD and adjacent non-tumor tissue samples were obtained from patients undergoing operations in the department of thoracic surgery, Jiangsu Cancer Hospital, from 2015 to 2018. None of the patients were previously treated with radiotherapy, chemotherapy or immunotherapy. All LUAD tissues were confirmed by the histopathological reports. The study was approved by the ethics committee of Jiangsu Cancer Hospital and written informed consent was obtained from all participants.

\section{Cell culture}

Human bronchial epithelial cell (HBE) and lung adenocarcinoma cell lines (A549, H1299, PC9 and H1975) were purchased from Shanghai Institutes for Biological Science (Shanghai, China). HBE and H1975 were cultivated in DMEM (KeyGene, Nanjing, China), while A549, H1299 and PC9 cells were cultivated in RPMI1640(KeyGene, Nanjing, China), all supplemented with 10\% FBS (KeyGene, Nanjing, China) and cultured in a standard $37^{\circ} \mathrm{C}$ incubator. For glucose starvation experiments, cells were cultured in medium with different concentrations of glucose.

\section{RNA extraction and qRT-PCR}

RNA was extracted from tissue samples or cultured cells with TRIzol reagent (Invitrogen, CA, USA). The PrimeScript RT Master Mix Perfect Real Time kit (Takara, Nanjing, China) was used to synthesize cDNA and qRT-PCR was performed with SYBR Select Master Mix (Takara, Nanjing, China). The expression levels of mRNA were normalized to ACTIN as reference genes. Primers used in this study are shown in Table S2.

\section{Transfection}

Human GAS6-AS1, GLUT1 and E2F1 CDNA were PCR-amplified and then cloned into the expression vector pcDND3.1 (RiboBio, Guangzhou, China). Transfection was carried out with Lipofectamine 3000 (Invitrogen, CA, USA) according to the manufacturer's instructions and empty vector plasmid was used as negative control. For experiments in vivo, GAS6-AS1 expression plasmids were packaged with pPACKH1 Lentivector Packaging Plasmid mix (System Biosciences, CA, USA) and were introduced into 293T cells. The liquid supernatant was transected into A549 cells and stable transfected cells were positively selected with puromycin (Thermo Fisher Scientific, Shanghai, China) for 4-6 weeks.

\section{Cell proliferation assays}

Cell proliferation assays were performed $24 \mathrm{~h}$ after transfection. For CCK8 viability assay, cells were seeded at a density of 2000 cells/well in a 96-well plate. 10\% CCK8 (Beyotime, Suzhou, China) was added to the well and the absorbance at $450 \mathrm{~nm}$ was measured every $24 \mathrm{~h}$. For 5-ethynyl-2'-deoxyuridine (EdU) assay, 8000 cells $/ 100 \mu \mathrm{L}$ were plated in 96-well plates. $100 \mu \mathrm{L} 50 \mu \mathrm{M}$ EdU solution (EdU Apollo®488 In Vitro Imaging Kit, RiboBio, Guangzhou, China) was incubated with cells. Edu were stained with red 
fluorescence and images were photographed by fluorescence microscope to count proportion of proliferating cells.

\section{Migration and invasion assays}

The migration and invasion assays were conducted with Transwell assay inserts ( $8 \mu M$ PET, 24-well Millicell) and Matrigel coated inserts (BD Biosciences, Bedford, USA), respectively. The upper chambers of the inserts were added with $200 \mu \mathrm{L}$ serum-free medium and the lower chambers were filled with $800 \mu \mathrm{L}$ medium containing $10 \%$ FBS. Totally $5 \times 10^{4}$ cells were added to the upper chambers and incubated for $24 \mathrm{~h}$ (migration) or $48 \mathrm{~h}$ (invasion). The migrated or invaded cells in the lower chambers were stained with crystal violet and counted.

\section{Tumor xenograft experiment}

The procedures for animal care and use were approved by Nanjing Medical University Animal Care Committee. 10 female nude mice (4-6 weeks old) were purchased from Nanjing Medical University School of Medicine's accredited animal facility and randomly divided into two groups. A549 cells stably expressing GAS6-AS1 or empty vector were injected in axilla subcutaneously of the mice. The volume of xenografts was measured every week using calipers ([length $\times$ width $\left.{ }^{2}\right] / 2$ ). All mice were euthanized 6 weeks after injection and tumors xenografts were harvested and weighed.

\section{Glucose uptake}

Glucose uptake was measured by Glucose Uptake Assay Kit (Abcam, Cambridge, UK). Cells were seeded in a 96-well plate at a density of 2000 cells/well and were starved in $100 \mu \mathrm{L}$ serum-free medium overnight to increase glucose uptake. Cells were then added with 2-DG and incubated for $20 \mathrm{~min}$ at $37^{\circ} \mathrm{C}$.

Exogenous 2-DG were removed by washing cells with PBS and cells were lysed with extraction buffer. The uptake of 2-DG was measured by colorimetric assay following the manufacturer's guidelines.

\section{Lactate and pyruvate measurement}

Lactate and pyruvate levels of cell supernatant were measured with an L-Lactate Assay Kit (Abcam, Cambridge, UK) and a Pyruvate Assay Kit (Abcam, Cambridge, UK) according to the manufacturer's instructions, respectively. The final results were normalized by total protein concentration.

\section{Extracellular Acidification Rate (ECAR)}

The Seahorse XF 24 Extracellular Flux Analyzer (Agilent, Beijing, China) was used to monitor ECAR of cells. Briefly, cells were seeded in a XF 24-well plate at a density of $1 \times 10^{4}$ per well and allowed to attach overnight. After serum starvation for $24 \mathrm{~h}$, ECAR was detected with a Seahorse XF Glycolysis Stress Test kit (Agilent, Beijing, China) according to the manufacturer's instructions. The measurements were normalized to total protein concentration.

\section{Western blotting}


Cells were lysed in RIPA buffer (KeyGEN, Nanjing, China) containing protease inhibitors PMSF (KeyGEN, Nanjing, China) and analyzed for total protein concentration. Western blotting was obtained utilizing 20$40 \mu \mathrm{g}$ of lysate protein according to the standard protocol. The following antibodies were used in this study: GLUT1 (Abcam, ab652, 1:1000 dilution), GLUT2 (Abcam, ab54460, 1:1000 dilution), GLUT3 (Abcam, ab41525, 1:1000 dilution), GLUT4 (Abcam, ab654, 1:1000 dilution), GLUT5 (Abcam, ab36057, 1:1000 dilution), HK2 (Cell Signaling Technology, 2867, 1:1000 dilution), ALDOC (Abcam, ab87122, 1:1000 dilution), ENO1 (Proteintech, 11204-1-AP, 1:1000 dilution), PKM (Abcam, ab38237, 1:1000 dilution), LDH (Cell Signaling Technology, 3582, 1:1000 dilution), ACTIN (Abcam, ab15265, 1:1000 dilution) and E2F1 (Santa Cruz Biotechnology, sc-251, 1:1000 dilution).

\section{Subcellular fractionation analysis}

Subcellular isolation of RNA was performed using a PARIS Kit (Invitrogen, CA, USA) according to the manufacturer's instructions. The isolated RNA was purified and further analyzed by qRT-PCR. GAPDH and U6 were used as cytoplasmic and nuclear controls, respectively.

\section{RNA immunoprecipitation (RIP)}

RNA immunoprecipitation (RIP) assay was carried out with a Magna RIP ${ }^{\mathrm{TM}}$ RNA-Binding Protein Immunoprecipitation Kit (Millipore, Massachusetts, USA) according to the manufacturer's instructions. Cells were lysed in the presence of protease and RNase inhibitors. Magnetic beads were then preincubated with IgG (Santa Cruz Biotechnology, sc-2025) or anti-E2F1 (Santa Cruz Biotechnology, sc-251). The prepared cell lysates underwent immunoprecipitation at $4{ }^{\circ} \mathrm{C}$ overnight and the co-precipitated RNAs were detected by qRT-PCR.

\section{Biotin-RNA pulldown assay}

mMESSAGE mMACHINE ${ }^{\text {TM }}$ T7 Transcription Kit (Invitrogen, CA, USA) was used for translation assays in vitro. Then, the targeted RNA was biotin-labeled with desthiobiotinylation using the Pierce RNA 3' End Desthiobiotinylation Kit (Invitrogen, CA, USA). Cells were collected and lysed by protein lysis buffer, while Streptavidin magnetic beads were used to capture the biotin-labeled RNA probe. RNA pull-down assays were performed with Pierce Magnetic RNA-Protein Pull-Down Kit (Invitrogen, CA, USA) according to the manufacturer's instructions. The pulldown extractions were eluted and subjected to western blotting analysis.

\section{Luciferase reporter assay}

GLUT1 promoter region containing E2F1 putative binding site (wild type) or mutant site (mutant type) was constructed into pGL3-based vectors which contains the firefly luciferase gene to establish two constructs, wt-GLUT1-luc and mut-GLUT1-luc. Overexpression constructs (empty vectors, E2F1 or GAS6AS1) were co-transfected with wt-GLUT1-luc and mut-GLUT1-luc, respectively. The cells were harvested to detect luciferase activity using the Dual-Luciferase reporter assay system (Promega, Madison, USA) $24 \mathrm{~h}$ after transfection. The ratio of firefly luciferase to renilla activity was calculated to measure luciferase activity. 


\section{Chromatin Immunoprecipitation (ChIP)}

ChIP was performed with a ChIP assay kit (Beyotime, Shanghai, China) according to the manufacturer's protocol. Briefly, A549 cells were cross-linked in 4\% paraformaldehyde and DNA was sheared by sonication to yield soluble chromatin. Cell lysates were incubated with protein A/G beads coated with anti-E2F1 (Santa Cruz Biotechnology, sc-251) or anti-mouse IgG (Santa Cruz Biotechnology, sc-2025). Immunoprecipitation was conducted at $4{ }^{\circ} \mathrm{C}$ overnight. Cross-linked DNA was eluted from the protein-DNA complex and further subjected to qRT-PCR.

\section{Statistical analysis}

Statistical analysis was performed using SPSS 22.0 software and GraphPad Prism 8.0. All values were presented as means \pm standard deviation (SD). Student's t-test was used to determine statistical differences between two groups. The chi-square test was used to analyze the relationship between GAS6AS1 expression and clinicopathological characteristics. All the repetitive experiments were repeated three times and $\mathrm{P}<0.05$ was considered significant.

\section{Results}

\section{Ectopic expression of GAS6-AS1 suppressed tumor progression in LUAD}

We analyzed GEO dataset (GSE56843) and GEPIA dataset to probe metabolism-related IncRNAs in LUAD. A microarray analysis of GSE56843 was performed to compare the gene expression profiles of A549 cells cultured with glucose and without glucose. Totally 4245 dysregulated genes were detected in LUAD cells induced by glucose starvation (screening criterion: adj.P.Val < 0.001, P.Value < 0.001, $|\mathrm{LogFC}|>1$ ). GEPIA dataset was searched to seek differentially expressed genes (screening criterion: $|\log 2 \mathrm{FC}|>1$, q-value < 0.01 ) and differential survival genes (screening criterion: Group Cutoff: Median, P-value < 0.01 , Top 500) in LUAD. Finally 13 genes were obtained by overlapping these results and two IncRNAs (LINC-PINT, GAS6AS1) were included (FigureS1A). The expression profile of candidate 13 genes in GSE56843 and GEPIA dataset were shown in FigureS1B and FigureS1C. LINC-PINT has been reported to function as a tumoursuppressor that exerts important regulatory roles in NSCLC progression by sponging miR-208a3p/PDCD4[21]. The role of GAS6-AS1 in lung cancer has not been identified and we select it as our research subject.

To elucidate the function of GAS6-AS1 in LUAD, plasmids were transfected into LUAD cells to overexpress GAS6-AS1. Confirmed by qRT-PCR, expression plasmids effectively manipulated the expression of GAS6AS1 in A549 and PC9 cells (Fig. 1A). Cell proliferation was analyzed using CCK-8 and EdU staining assays, and results showed that cell proliferative capability was remarkably attenuated by overexpression of GAS6-AS1 (Fig. 1B-E). Transwell and Matrigel assays were used to test the migration and invasion ability of LUAD cells, respectively. The results indicated that ectopic expression of GAS6-AS1 inhibited 
A549 cells and PC9 cells to migrate and invade (Fig. 1F-G). And xenograft tumor model was used to evaluate the tumor-suppressive role of GAS6-AS1 in vivo. Empty vector $(n=6)$ and GAS6-AS1-transfected cells $(n=6)$ were injected in axilla subcutaneously of nude mice. Tumor nodules were resected after injection of 6 weeks and the volume and weight of tumors were recorded (Fig. 1H). As shown in Fiugre1I and Fig. 1J, tumors of GAS6-AS1-transfected group grew slowly with smaller size and lighter weight. These results suggested that GAS6-AS1 exerted tumor-suppressive roles in LUAD both in vitro and in vivo.

\section{GAS6-AS1 impaired glucose metabolism reprogramming in LUAD cells}

GAS6-AS1 was a IncRNA abnormally expressed in A549 cells cultured without glucose (FigureS1B). It has been reported in 1988 that glucose starvation is required for insulin stimulation of glucose uptake and metabolism in cultured microvascular endothelial cells[22]. Moreover, cell growth and survival depend on a delicate balance between energy production and synthesis of metabolites, and energy stress is closely linked to metabolic alterations[23]. In tumor cells, glucose metabolism reprogramming will appear during energy stress to guarantee the energy supply and survival, manifesting that cancer cells uptake more glucose and produce more lactate and pyruvate (Warburg effect) [24]. We performed Gene_Ontology enrichment analysis on genes highly co-expressed with GAS6-AS1 and results suggested that these genes might participate in biological process of "regulation of transcription, DNA-templated" and "carboxylic acid metabolic process" (Fig. 2A), and molecular function of "protein binding" and "carboxylyase activity" (Fig. 2B). Based on these theories and predictions, we intended to study the role of GAS6AS1 in glucose metabolism reprogramming of LUAD cells. It turned out that ectopic expression of GAS6AS1 inhibited glucose consumption of A549 and PC9 cells (Fig. 2C). And the production of lactate and pyruvate in LUAD cells were decreased after overexpression of GAS6-AS1 (Fig. 2D-E). In addition, Seahorse analysis revealed decreased extracellular acidification rate (ECAR) in A549 and PC9 cells by ectopic expression of GAS6-AS1 (Figre2F-G). These results implied that GAS6-AS1 could impair glucose metabolism reprogramming of LUAD cells.

\section{GAS6-AS1 exerted tumor-suppressive roles via regulating expression of glucose transporter GLUT1}

Glucose metabolism reprogramming of cancer cells is driven by a number of specific enzymes, including GLUT1-5 (glucose transporter1-5, SLC2A1-5), HK2 (hexokinase 2), ALDOC (aldolase C), ENO1 (enolase 1), PKM (pyruvate kinase) and LDH (lactate dehydrogenase) (Fig. 3A)[25]. Real-time RT-PCR and western blotting were performed to explore the influence of GAS6-AS1 on these metabolism-related enzymes. Results showed that the mRNA and protein level of GLUT1 (glucose transporter1, also known as SLC2A1) were significantly downregulated after overexpression of GAS6-AS1 (Fig. 3B-C). GLUT1 belongs to the glucose transporter family and regulates glucose transport across the cell membrane[26]. Increasing studies indicate that GLUT1 participates in tumor progression and energy metabolism reprogramming in multiple cancer types[27-29]. To verify whether the function of GAS6-AS1 was dependent on GLUT1, we 
performed rescue experiments by transfecting expression plasmids of GLUT1 into GAS6-AS1overexpressed A549 cells. CCK8, Transwell and Matrigel assays revealed that GLUT1 restored the inhibition effect of GAS6-AS1 on proliferation, migration and invasion (Fig. 3D-F). Furtherly, glucose consumption, lactate production, pyruvate production and extracellular acidification rate (ECAR) were detected. Consistently, ectopic expression of GLUT1 restored the inhibition effect of GAS6-AS1 on glucose metabolism reprogramming in A549 cells (Fig. 3G-J). These results suggested that GAS6-AS1 suppressed tumor progression and glucose metabolism reprogramming via downregulating glucose transporter GLUT1.

\section{GAS6-AS1 interacted with E2F1}

As the subcellular localization of IncRNAs is crucial for their functional biology, nucleo-cytoplasmic separation assays were performed and results showed that GAS6-AS1 was mainly located in the nucleus (Fig. 4A). Nuclear is the place for transcription and our previous analysis indicated that GAS6-AS1 might participate in biological process of "regulation of transcription, DNA-templated" (Fig. 2A). In recent years, multiple IncRNAs were found to regulate the transcription of target genes via binding with specific transcription factors[30-32]. Through searching the LncMap datasets, a cluster of transcription factors were predicted to interact with GAS6-AS1 (Fig. 4B). Among these transcription factors, we noticed that E2F1 had been reported to enhance glycolysis and reprogram energy metabolism in cancer cells[33]. And Albert Tauler also found that E2F1 induced mTORC1 activation to regulate glucose metabolism[34]. Moreover, TF ChIP-seq data of ChIPBase (http://rna.sysu.edu.cn/chipbase/) showed that GLUT1 (SLC2A1) gene was predicted as a direct target of E2F1 in HeLa-S3 cells (Supplementary FigureS2A). We suspected that long coding RNA GAS6-AS1 might regulate the expression of GLUT1 via interacting with transcription factor E2F1. To validate our hypothesis, RNA immunoprecipitation (RIP) assay was performed in A549 cells and results indicated that E2F1 recruitment the RNA fragment of GAS6-AS1 (Fig. 4C). Western blot analysis of proteins extracted from GAS6-AS1 pull-down assay revealed that E2F1 protein specifically binds to the sense sequence of GAS6-AS1, but not to the antisense control (Fig. 4D-E). Additionally, the mRNA and protein expression of E2F1 were not altered after overexpression of GAS6AS1 (Fig. 4F-G). Furtherly, we used catRAPID tools to analyze the interaction region of GAS6-AS1 with E2F1 (Fig. 4H) and results showed that E2F1 might bind to nucleotides 526-728nt of GAS6-AS1 with high propensities (Fig. 4I). Meanwhile, the secondary structure of GAS6-AS1 was predicted by online database AnnoLnc (Fig. 4J). We next constructed a series of GAS6-AS1 truncations to map its binding fragment with E2F1 (Fig. 4K). Verified by RNA pulldown assays, fragment of GAS6-AS1 (526-728nt) was responsible for its interaction with E2F1 (Fig. 4L). These data indicated that GAS6-AS1 specifically bound to transcription factor E2F1.

\section{GAS6-AS1 suppressed E2F1-mediated transcription of GLUT1}


Transcription factors regulated the expression of target genes through binding with specific DNA sequences. Predicted by Jaspar database, we found that E2F1 potentially bound to the promoter region of GLUT1 (Supplementary FigureS2B). To prove that GLUT1 is a transcriptional target of E2F1 and the regulation can be repressed by GAS6-AS1, luciferase vectors consisting of wild-type or mutant GLUT1 promoters were constructed and transfected into A549 cells (Fig. 5A). The luciferase assay revealed that E2F1 stimulated the wild-type GLUT1 promoter activity as indicated by increased luciferase activity, but had no effect on mutant-type GLUT1 promoter activity. Moreover, GAS6-AS1 suppressed the increase of luciferase activity induced by E2F1 (Fig. 5B). ChIP assay was furtherly performed and results suggested that E2F1 was bound to GLUT1 promoter and the interaction was repressed by GAS6-AS1 (Fig. 5C). In addition, the enforced expression of E2F1 significantly upregulated the mRNA and protein levels of GLUT1, of which could also be restored by GAS6-AS1 (Fig. 5D-E). These results suggested that E2F1 directly bound to promoter region of GLUT1 to activate its transcription, and GAS6-AS1 could inhibit the process. Consistently with these results, the expression of E2F1 was positively correlated with GLUT1 in LUAD while GAS6-AS1 was negatively correlated with GLUT1 (Fig. 5F-G).

\section{GAS6-AS1 was clinically relevant in LUAD}

To confirm the expression pattern of GAS6-AS1 in LUAD, we firstly searched the CCLE database and results showed that the expression of GAS6-AS1 in NSCLC cells (lung_NSC) were at a relatively low level (Fig. 6A). Furtherly, we performed qRT-PCR assay on 80 paired LUAD tissues and adjacent normal tissues and found that GAS6-AS1 was significantly lowly expressed in LUAD tissues (Fig. 6B). Moreover, patients of advanced T stages (T2-T4) exerted lower expression of GAS6-AS1 than patients of early T stages (T1) (Fig. 6C). Additionally, we divided the 80 LUAD samples into GAS6-AS1 low expression group (40 cases) and high expression group (40 cases) to investigate the clinical significance of GAS6-AS1 in LUAD. Results suggested that the expression of GAS6-AS1 correlated with tumor size, lymph node metastasis, cancer distant metastasis and TNM stage of LUAD tissues (Fig. 6D).The expression of GAS6-AS1 in common LUAD cell lines was also detected by qRT-PCR and we found that GAS6-AS1 was lowly expressed in 4 LUAD cell lines compared with normal human bronchial epithelial cell (HBE) (Fig. 6E). Furtherly, GEPIA dataset was explored to analyze the clinical correlation of GAS6-AS1 in LUAD. Results showed that GAS6-AS1 was lowly expressed in LUAD tissues (Fig. 6F) and the expression of GAS6-AS1 in LUAD correlated with tumor stage (Fig. 6G), overall survival (Fig. 6H) and disease free survival (Fig. 6I) of patients. Collectively, these results indicated that GAS6-AS1 was downregulated in LUAD tissues and correlated with tumor stages and survival of patients.

\section{Discussion}

Increased aerobic glycolysis even in the abundance of oxygen, or the "Warburg effect", has been recognized as an emerging hallmark of cancer. Distinguished from healthy cells, cancer cells exerted glucose metabolism remodeling, characterized by consuming glucose and excrete lactate at a significantly higher rate, and synthesizing ATP by producing lactic acid as the end product[35]. These metabolic changes benefit cancer cells in multiple aspects. The switching to oxygen-independent 
glycolysis make cancer cells more resistant to hypoxia condition associated with tumor growth. And the glycolytic intermediates fuel several biosynthetic pathways that produce de novo nucleotides, lipids, amino acids, and NADPH. Moreover, the increased production of lactic acid lower the $\mathrm{pH}$ of extracellular microenvironment and support the invasion and metastasis of cancer cells[36]. The pivotal molecular mechanism for the initiation and development of metabolic remodeling still remain unclear. To target cancer cells from the point of glucose metabolism might provide attractive and effective therapeutic approaches.

Lung cancer is the leading cause of cancer-related death with limited curative treatment options, and extensive studies have revealed the reprogramming of glucose metabolism in lung cancer[37, 38]. The alterations of oncogenes and tumor suppressor genes, leading to activation or inhibition of signaling pathways and transcriptional networks, were reported to participate in the glucose metabolism reprogramming of lung cancer[39]. However, the potential involvement of IncRNAs is poorly defined in glucose metabolism reprogramming of lung cancer. In our study, utilizing publicly available IncRNA expression profiling data in A549 cells under glucose starvation and integrating analyses of GEPIA data, we screened a metabolic-related IncRNA GAS6-AS1 in LUAD. GAS6-AS1, which is located at chromosome $13 q 34$, is an antisense transcript of GAS6. Previous research revealed that low expression of GAS6-AS1 predicted a poor prognosis in patients with NSCLC[40]. And GAS6-AS1 was identified to play vital roles in the progression of gastric cancer and hepatocellular carcinoma[41, 42]. In our study, we further found that the expression of GAS6-AS1 was frequently downregulated in LUAD tissues and clinical analysis reveals that downregulation of GAS6-AS1 correlated with advanced tumor stage and poor prognosis of LUAD patients. Moreover, ectopic expression of GAS6-AS1 inhibited tumor growth and glucose metabolism remodeling in LUAD cells. Our results unraveled an important role of GAS6-AS1 in the cancer development and glucose metabolism of LUAD.

The reprogramming of cancer metabolism is driven by several key enzymes. Glucose transporters (GLUTs) are membrane proteins that transport glucose from the capillaries into cells and mediate the first step for cellular glucose usage. Five main classical glucose transporters (GLUT1, GLUT2, GLUT3, GLUT4, GLUT5) with different affinities for glucose have been reported to function in glucose uptake for glycolysis of cancers[43]. The phosphorylation of glucose, a crucial step in cellular metabolism, is catalysed by Hexokinases II (HK2) and HK2 is considered as the hub in the regulation of cancer cell glycolysis[44]. Aldolase C (ALDOC) is a glycolytic enzyme that catalyzes the reversible aldol cleavage of fructose-1,6-biphosphate (F1,6BP) to glyceraldehyde-3-phosphate (G3P)[45]. Enolase 1 (ENO1) transforms 2-phospho-D-glycerate (2-PGA) into phosphoenolpyruvate (PEP) during glycolysis[46], followed by PKM transferring of a phosphoryl group from phosphoenolpyruvate (PEP) to ADP, generating ATP and pyruvate[47]. And lactate dehydrogenase (LDH), is responsible for the conversion of L-lactate and NAD to pyruvate and NADH in the final step of anaerobic glycolysis[48]. We performed qRT-PCR and western blotting to detect the influence of GAS6-AS1 on these enzymes and results indicated that GAS6AS1 remarkably decreased the expression of GLUT1 in both mRNA and protein levels. GLUT1 (also known as solute carrier family 2 member 1, SLC2A1) is a key rate-limiting factor in the transport of glucose in cancer cells and plays a critical role in glycolysis and tumor progression in multiple cancer 
types[49-51]. Numerous IncRNAs have been proved to regulate GLUT1 in various ways. HOX transcript antisense RNA (HOTAIR) influences glucose metabolism through GLUT1 upregulation via mTOR signaling[52]. LncRNA-p23154 promotes glycolysis in oral squamous cell carcinoma by binding to the promoter region of miR-378a-3p, which represses GLUT1 expression by targeting to its 3'UTR directly[53]. SLC2A1-AS1 regulates aerobic glycolysis and progression in hepatocellular carcinoma by competitively binding to STAT3 and resulting in inactivation of the FOXM1/GLUT1 axis[54]. Consistent with these studies, we showed that GAS6-AS1 exerted its role in tumor progression and glucose metabolism in LUAD via downregulating GLUT1.

Mechanistic investigations revealed that GAS6-AS1 could bind to transcription factor E2F1, but not affect E2F1 expression. Previous studies have shown that E2F1 coactivated by KDM4A regulates the PDKdependent metabolic switch between mitochondrial oxidation and glycolysis[55]. Besides, E2F1 was reported to significantly enhance glucose uptake and lactate production in bladder and prostate cancer cell lines[33]. LncRNAs have been identified to function as master regulators for gene expression in many ways, and one is to interact with transcription factor and thus transcriptionally regulating downstream genes[56]. For instance, IncRNA CAIF directly binds to p53 protein and blocks p53-mediated myocardin transcription in cardiomyocyte autophagy[57] and PAXIP1-AS1 facilitates cell invasion and angiogenesis of glioma by recruiting transcription factor ETS1 to upregulate KIF14 expression[58]. Likewise, our results suggested that GAS6-AS1 integrated with E2F1 and suppressed E2F1-mediated transcription of GLUT1.

\section{Conclusion}

Taken together, our study first identified IncRNA GAS6-AS1 as a suppressor in tumor progression and glucose metabolism reprogramming of LUAD. The downregulation of GAS6-AS1 correlated with tumor stage and overall survival of LUAD patients. And GAS6-AS1 exerted its biological role through binding with E2F1 and suppressing the E2F1-mediated transcription of glucose transporter, GLUT1 (Fig. 7). Therefore, GAS6-AS1 may serve as potential diagnostic and prognostic biomarker for LUAD patients, and targeting the GAS6-AS1/E2F1/GLUT1 axis may present new perspectives in the prevention or treatment of LUAD.

\section{Abbreviations}

IncRNA: long non coding RNA; LUAD: lung adenocarcinoma; ECAR: extracellular acidification rate; RIP: RNA immunoprecipitation; ChIP: Chromatin Immunoprecipitation; ATP: adenosine triphosphate; ADP: Adenosine diphosphate; NSCLC: non-small cell lung cancer; SCLC: small-cell lung cancer; GLUT: glucose transporter; HK2: hexokinase 2; ALDOC: aldolase C; ENO1: enolase 1; PKM: pyruvate kinase; LDH: lactate dehydrogenase; NADPH: nicotinamide adenine dinucleotide phosphate; F1,6BP: fructose-1,6-biphosphate; G3P: glyceraldehyde-3-phosphate; 2-PGA: 2-phospho-D-glycerate; PEP: phosphoenolpyruvate; HOTAIR: HOX transcript antisense RNA

\section{Declarations}




\section{Ethics approval and consent to participate}

The study was approved by the ethics committee of Jiangsu Cancer Hospital and written informed consent was obtained from all participants.

\section{Consent for publication}

Consent for publication has been obtained from all authors.

\section{Availability of data and material}

The datasets used and/or analyzed during the current study are available from the corresponding author on reasonable request.

\section{Competing interests}

The authors report no conflicts of interest in this work.

\section{Funding}

This research was supported by National Natural Science Foundation for Youth of China (No. 81902354), the National Natural Science Foundation of China (81672869), Jiangsu Provincial Medical Outstanding Talent (Lin Xu), Jiangsu Provincial Medical Youth Talent (Binhui Ren, QNRC2016657), the talents program of Jiangsu cancer hospital YC201814, the "333" talent project: BRA2019325, Science and Technology program of Xu Zhou (No. KC18037) and Xuzhou Clinical Technology Key Research Project (2019GG021).

\section{Author contributions}

Lin Xu, Yi Shen, and Binhui Ren designed and supervised the study. Jing Luo, Huishan Wang and Li Wang performed most of the experiments and wrote the manuscript. Gaoming Wang was responsible for statistical analysis. Yu Yao helped to conduct experiments in vivo. Kai Xie and Xiaokun Li performed some mechanistic experiments and edited the manuscript. All authors read and approved the final version of the manuscript.

\section{Acknowledgements}

The authors thank NanJing XinJia Medical Technology Co. Ltd for providing technical platform support.

\section{References}

1. DeBerardinis RJ, Chandel NS. Fundamentals of cancer metabolism. Sci Adv. 2016;2:e1600200.

2. Cantor JR, Sabatini DM. Cancer cell metabolism: one hallmark, many faces. Cancer Discov. 2012;2:881-98. 
3. Vander Heiden MG, Cantley LC, Thompson CB. Understanding the Warburg effect: the metabolic requirements of cell proliferation. Science. 2009;324:1029-33.

4. Boroughs LK, DeBerardinis RJ. Metabolic pathways promoting cancer cell survival and growth. Nat Cell Biol. 2015;17:351-9.

5. Khorkova O, Hsiao J, Wahlestedt C. Basic biology and therapeutic implications of IncRNA. Adv Drug Deliv Rev. 2015;87:15-24.

6. Charles Richard JL, Eichhorn PJA. Platforms for Investigating LncRNA Functions. SLAS Technol. 2018;23:493-506.

7. Bhan A, Soleimani M, Mandal SS. Long Noncoding RNA and Cancer: A New Paradigm. Cancer Res. 2017;77:3965-81.

8. Wang J, Zhang X, Chen W, Hu X, Li J, Liu C. Regulatory roles of long noncoding RNAs implicated in cancer hallmarks. Int J Cancer. 2020;146:906-16.

9. Siegel RL, Miller KD, Jemal A. Cancer statistics, 2020. CA Cancer J Clin. 2020;70:7-30.

10. Herbst RS, Heymach JV, Lippman SM. Lung cancer. N Engl J Med. 2008;359:1367-80.

11. Fan TW, Lane AN, Higashi RM, Farag MA, Gao H, Bousamra M, Miller DM. Altered regulation of metabolic pathways in human lung cancer discerned by (13)C stable isotope-resolved metabolomics (SIRM). Mol Cancer. 2009;8:41.

12. Cancer Genome Atlas Research N. Comprehensive molecular profiling of lung adenocarcinoma. Nature. 2014;511:543-50.

13. Kerr EM, Martins CP. Metabolic rewiring in mutant Kras lung cancer. FEBS J. 2018;285:28-41.

14. Jozwiak P, Lipinska A. [The role of glucose transporter 1 (GLUT1) in the diagnosis and therapy of tumors]. Postepy Hig Med Dosw (Online). 2012;66:165-74.

15. Tang Z, Li C, Kang B, Gao G, Li C, Zhang Z. GEPIA: a web server for cancer and normal gene expression profiling and interactive analyses. Nucleic Acids Res. 2017;45:W98-102.

16. Huang DW, Sherman BT, Tan Q, Kir J, Liu D, Bryant D, Guo Y, Stephens R, Baseler MW, Lane HC, Lempicki RA. DAVID Bioinformatics Resources: expanded annotation database and novel algorithms to better extract biology from large gene lists. Nucleic Acids Res. 2007;35:W169-75.

17. Li Y, Li L, Wang Z, Pan T, Sahni N, Jin X, Wang G, Li J, Zheng X, Zhang Y, Xu J, Yi S, Li X. LncMAP: Pan-cancer atlas of long noncoding RNA-mediated transcriptional network perturbations. Nucleic Acids Res. 2018;46:1113-23.

18. Hou M, Tang X, Tian F, Shi F, Liu F, Gao G. AnnoLnc: a web server for systematically annotating novel human IncRNAs. BMC Genom. 2016;17:931.

19. Agostini F, Zanzoni A, Klus P, Marchese D, Cirillo D, Tartaglia GG. catRAPID omics: a web server for large-scale prediction of protein-RNA interactions. Bioinformatics. 2013;29:2928-30.

20. Mathelier A, Fornes O, Arenillas DJ, Chen CY, Denay G, Lee J, Shi W, Shyr C, Tan G, Worsley-Hunt R, Zhang AW, Parcy F, Lenhard B, Sandelin A, Wasserman WW. JASPAR 2016: a major expansion and 
update of the open-access database of transcription factor binding profiles. Nucleic Acids Res. 2016;44:D110-5.

21. Zhang L, Hu J, Li J, Yang Q, Hao M, Bu L. Long noncoding RNA LINC-PINT inhibits non-small cell lung cancer progression through sponging miR-218-5p/PDCD4. Artif Cells Nanomed Biotechnol. 2019;47:1595-602.

22. Gerritsen ME, Burke TM, Allen LA. Glucose starvation is required for insulin stimulation of glucose uptake and metabolism in cultured microvascular endothelial cells. Microvasc Res. 1988;35:153-66.

23. Bezawork-Geleta A, Wen H, Dong L, Yan B, Vider J, Boukalova S, Krobova L, Vanova K, Zobalova R, Sobol M, Hozak P, Novais SM, Caisova V, Abaffy P, Naraine R, Pang Y, Zaw T, Zhang P, Sindelka R, Kubista M, Zuryn S, Molloy MP, Berridge MV, Pacak K, Rohlena J, Park S, Neuzil J. Alternative assembly of respiratory complex II connects energy stress to metabolic checkpoints. Nat Commun. 2018;9:2221.

24. Li J, Huang Q, Long X, Guo X, Sun X, Jin X, Li Z, Ren T, Yuan P, Huang X, Zhang H, Xing J. Mitochondrial elongation-mediated glucose metabolism reprogramming is essential for tumour cell survival during energy stress. Oncogene. 2017;36:4901-12.

25. Doherty JR, Cleveland JL. Targeting lactate metabolism for cancer therapeutics. J Clin Invest. 2013;123:3685-92.

26. Deng D, Xu C, Sun P, Wu J, Yan C, Hu M, Yan N. Crystal structure of the human glucose transporter GLUT1. Nature. 2014;510:121-5.

27. Xiao H, Wang J, Yan W, Cui Y, Chen Z, Gao X, Wen X, Chen J. GLUT1 regulates cell glycolysis and proliferation in prostate cancer. Prostate. 2018;78:86-94.

28. Chen J, Cao L, Li Z, Li Y. SIRT1 promotes GLUT1 expression and bladder cancer progression via regulation of glucose uptake. Hum Cell. 2019;32:193-201.

29. Liu YX, Feng JY, Sun MM, Liu BW, Yang G, Bu YN, Zhao M, Wang TJ, Zhang WY, Yuan HF, Zhang XD. Aspirin inhibits the proliferation of hepatoma cells through controlling GLUT1-mediated glucose metabolism. Acta Pharmacol Sin. 2019;40:122-32.

30. Zhang E, He X, Zhang C, Su J, Lu X, Si X, Chen J, Yin D, Han L. and De W. A novel long noncoding RNA HOXC-AS3 mediates tumorigenesis of gastric cancer by binding to YBX1. Genome Biol. 2018;19:154.

31. Peng Z, Wang J, Shan B, Li B, Peng W, Dong Y, Shi W, Zhao W, He D, Duan M, Cheng Y, Zhang C, Duan C. The long noncoding RNA LINC00312 induces lung adenocarcinoma migration and vasculogenic mimicry through directly binding YBX1. Mol Cancer. 2018;17:167.

32. Jiang H, Li T, Qu Y, Wang X, Li B, Song J, Sun X, Tang Y, Wan J, Yu Y, Zhan J, Zhang H. Long noncoding RNA SNHG15 interacts with and stabilizes transcription factor Slug and promotes colon cancer progression. Cancer Lett. 2018;425:78-87.

33. Wu M, Seto E, Zhang J. E2F1 enhances glycolysis through suppressing Sirt6 transcription in cancer cells. Oncotarget. 2015;6:11252-63. 
34. Almacellas E, Pelletier J, Manzano A, Gentilella A, Ambrosio S, Mauvezin C, Tauler A. Phosphofructokinases Axis Controls Glucose-Dependent mTORC1 Activation Driven by E2F1. iScience. 2019;20:434-48.

35. Anastasiou D. Tumour microenvironment factors shaping the cancer metabolism landscape. $\mathrm{Br} \mathrm{J}$ Cancer. 2017;116:277-86.

36. Jozwiak P, Forma E, Brys M, Krzeslak A. O-GlcNAcylation and Metabolic Reprograming in Cancer. Front Endocrinol (Lausanne). 2014;5:145.

37. Nakamoto Y, Zasadny KR, Minn H, Wahl RL. Reproducibility of common semi-quantitative parameters for evaluating lung cancer glucose metabolism with positron emission tomography using 2-deoxy-2-[18F]fluoro-D-glucose. Mol Imaging Biol. 2002;4:171-8.

38. Momcilovic M, Bailey ST, Lee JT, Zamilpa C, Jones A, Abdelhady G, Mansfield J, Francis KP, Shackelford DB. Utilizing 18F-FDG PET/CT Imaging and Quantitative Histology to Measure Dynamic Changes in the Glucose Metabolism in Mouse Models of Lung Cancer. J Vis Exp 2018.

39. Vanhove K, Graulus GJ, Mesotten L, Thomeer M, Derveaux E, Noben JP, Guedens W, Adriaensens P. The Metabolic Landscape of Lung Cancer: New Insights in a Disturbed Glucose Metabolism. Front Oncol. 2019;9:1215.

40. Han L, Kong R, Yin DD, Zhang EB, Xu TP, De W, Shu YQ. Low expression of long noncoding RNA GAS6-AS1 predicts a poor prognosis in patients with NSCLC. Med Oncol. 2013;30:694.

41. Zhang $P$, Dong $Q$, Zhu $H$, Li S, Shi L, Chen X. Long non-coding antisense RNA GAS6-AS1 supports gastric cancer progression via increasing GAS6 expression. Gene. 2019;696:1-9.

42. Ai J, Sun J, Zhou G, Zhu T, Jing L. Long non-coding RNA GAS6-AS1 acts as a ceRNA for microRNA585, thereby increasing EIF5A2 expression and facilitating hepatocellular carcinoma oncogenicity. Cell Cycle. 2020;19:742-57.

43. Ancey PB, Contat C, Meylan E. Glucose transporters in cancer - from tumor cells to the tumor microenvironment. FEBS J. 2018;285:2926-43.

44. Smith TA. Mammalian hexokinases and their abnormal expression in cancer. Br J Biomed Sci. 2000;57:170-8.

45. Buono P, Cassano S, Alfieri A, Mancini A, Salvatore F. Human aldolase $C$ gene expression is regulated by adenosine 3',5'-cyclic monophosphate (cAMP) in PC12 cells. Gene. 2002;291:115-21.

46. Chen JM, Chiu SC, Chen KC, Huang YJ, Liao YA, Yu CR. Enolase 1 differentially contributes to cell transformation in lung cancer but not in esophageal cancer. Oncol Lett. 2020;19:3189-96.

47. Xiong Y, Lei QY, Zhao S, Guan KL. Regulation of glycolysis and gluconeogenesis by acetylation of PKM and PEPCK. Cold Spring Harb Symp Quant Biol. 2011;76:285-9.

48. Yeung C, Gibson AE, Issaq SH, Oshima N, Baumgart JT, Edessa LD, Rai G, Urban DJ, Johnson MS, Benavides GA, Squadrito GL, Yohe ME, Lei H, Eldridge S, Hamre J 3rd, Dowdy T, Ruiz-Rodado V, Lita A, Mendoza A, Shern JF, Larion M, Helman LJ, Stott GM, Krishna MC, Hall MD, Darley-Usmar V, Neckers LM, Heske CM. Targeting Glycolysis through Inhibition of Lactate Dehydrogenase Impairs Tumor Growth in Preclinical Models of Ewing Sarcoma. Cancer Res. 2019;79:5060-73. 
49. Koh YW, Park SY, Hyun SH, Lee SJ. Associations Between PET Textural Features and GLUT1 Expression, and the Prognostic Significance of Textural Features in Lung Adenocarcinoma. Anticancer Res. 2018;38:1067-71.

50. Khabaz MN, Qureshi IA, Al-Maghrabi JA. GLUT 1 expression is a supportive mean in predicting prognosis and survival estimates of endometrial carcinoma. Ginekol Pol. 2019;90:582-8.

51. Sawayama H, Ogata Y, Ishimoto T, Mima K, Hiyoshi Y, Iwatsuki M, Baba Y, Miyamoto Y, Yoshida N, Baba H. Glucose transporter 1 regulates the proliferation and cisplatin sensitivity of esophageal cancer. Cancer Sci. 2019;110:1705-14.

52. Wei S, Fan Q, Yang L, Zhang X, Ma Y, Zong Z, Hua X, Su D, Sun H, Li H, Liu Z. Promotion of glycolysis by HOTAIR through GLUT1 upregulation via mTOR signaling. Oncol Rep. 2017;38:1902-8.

53. Wang Y, Zhang X, Wang Z, Hu Q, Wu J, Li Y, Ren X, Wu T, Tao X, Chen X, Li X, Xia J, Cheng B. LncRNAp23154 promotes the invasion-metastasis potential of oral squamous cell carcinoma by regulating Glut1-mediated glycolysis. Cancer Lett. 2018;434:172-83.

54. Shang R, Wang M, Dai B, Du J, Wang J, Liu Z, Qu S, Yang X, Liu J, Xia C, Wang L, Wang D, Li Y. Long noncoding RNA SLC2A1-AS1 regulates aerobic glycolysis and progression in hepatocellular carcinoma via inhibiting the STAT3/FOXM1/GLUT1 pathway. Mol Oncol. 2020;14:1381-96.

55. Wang LY, Hung CL, Chen YR, Yang JC, Wang J, Campbell M, Izumiya Y, Chen HW, Wang WC, Ann DK, Kung HJ. KDM4A Coactivates E2F1 to Regulate the PDK-Dependent Metabolic Switch between Mitochondrial Oxidation and Glycolysis. Cell Rep. 2016;16:3016-27.

56. Long Y, Wang X, Youmans DT, Cech TR. How do IncRNAs regulate transcription? Sci Adv. 2017;3:eaao2110.

57. Liu CY, Zhang YH, Li RB, Zhou LY, An T, Zhang RC, Zhai M, Huang Y, Yan KW, Dong YH, Ponnusamy M, Shan C, Xu S, Wang Q, Zhang YH, Zhang J, Wang K. LncRNA CAIF inhibits autophagy and attenuates myocardial infarction by blocking p53-mediated myocardin transcription. Nat Commun. 2018;9:29.

58. Xu H, Zhao G, Zhang Y, Jiang H, Wang W, Zhao D, Yu H, Qi L. Long non-coding RNA PAXIP1-AS1 facilitates cell invasion and angiogenesis of glioma by recruiting transcription factor ETS1 to upregulate KIF14 expression. J Exp Clin Cancer Res. 2019;38:486.

\section{Figures}


A

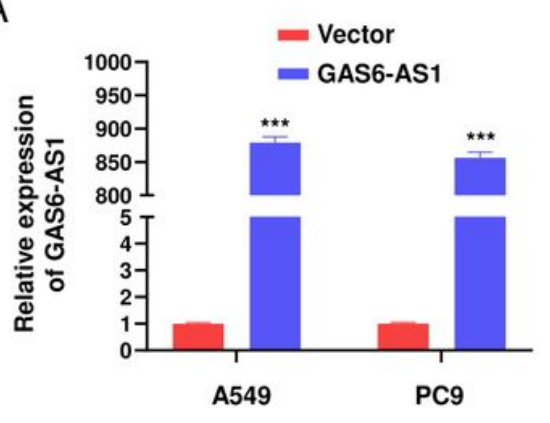

D

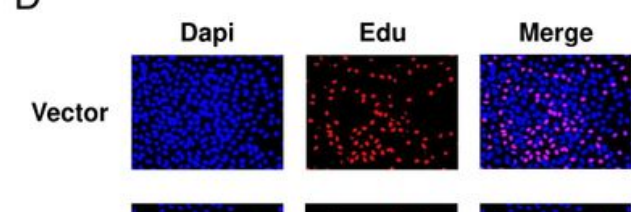

GAS6-AS1
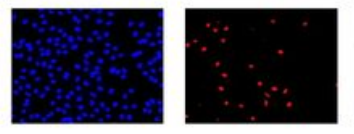

A549

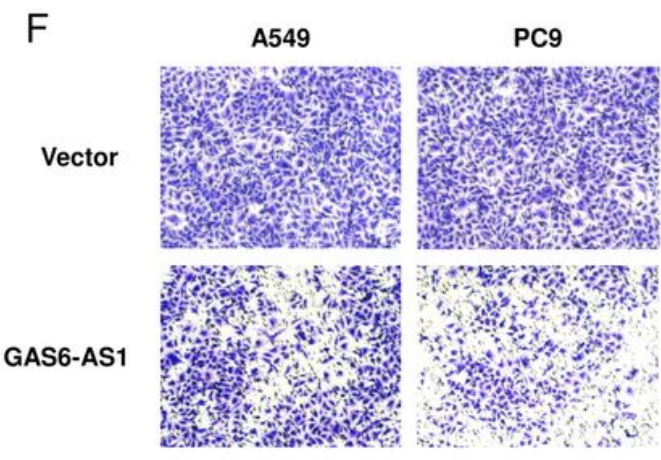

Migration

$\mathrm{H}$

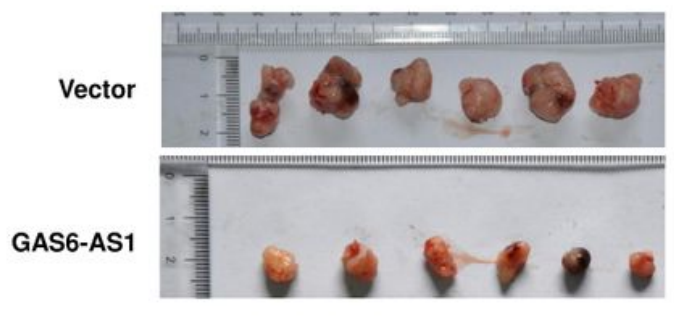

A549
B
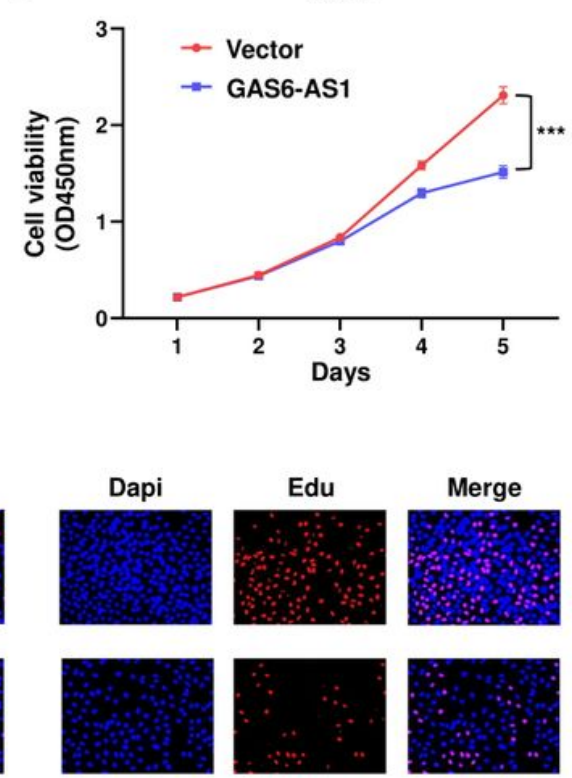

PC9

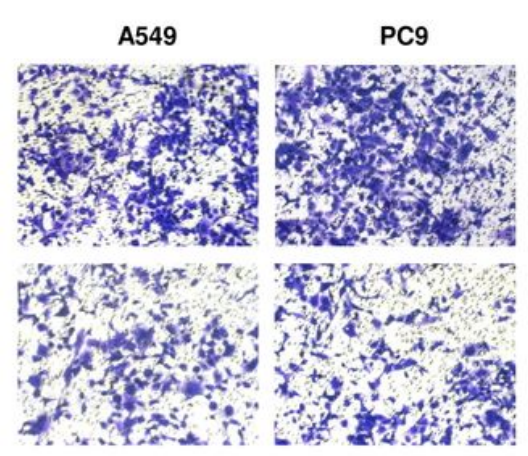

Invasion

I

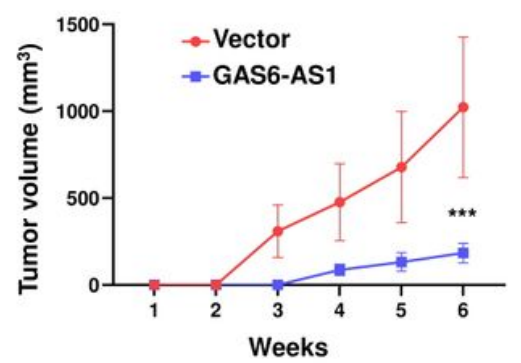

E
C

PC9
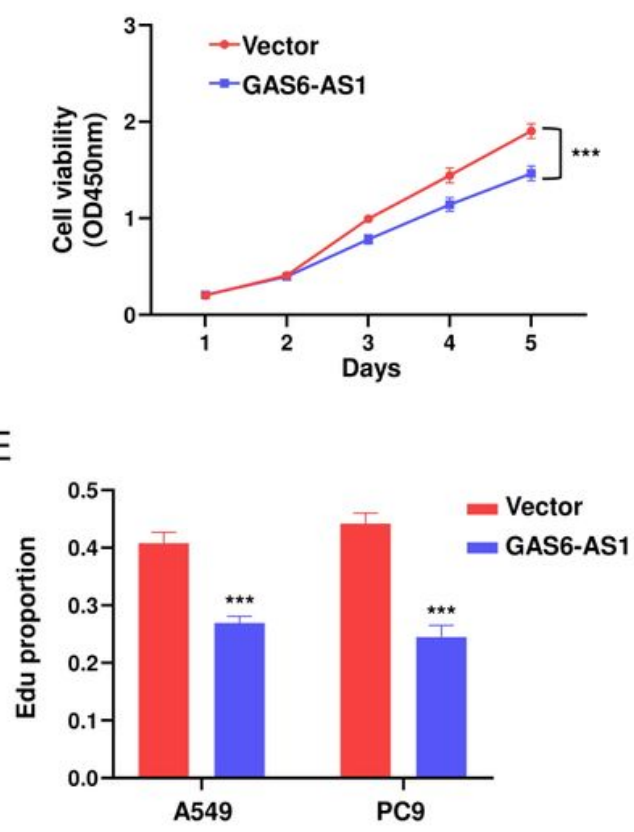

G

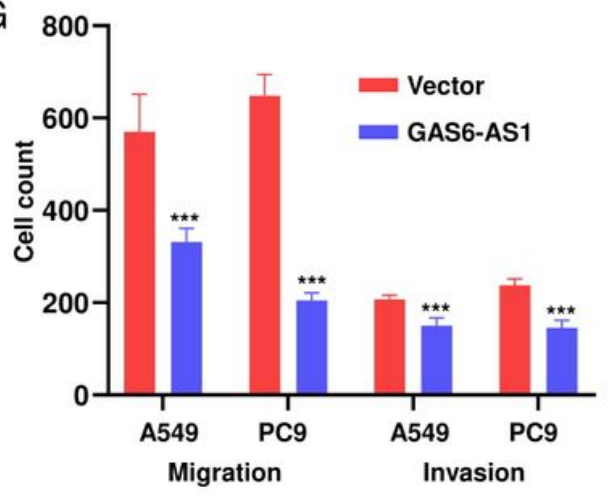

J

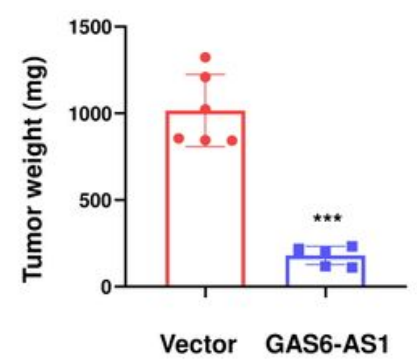

\section{Figure 1}

GAS6-AS1 functioned as a tumor suppressor function in LUAD. A. GAS6-AS1 was overexpressed in A549 and PC9 cells after transfection of overexpression plasmid of GAS6-AS1. B-C. CCK8 assays were used to detect cell proliferation of A549 cells and PC9 cells after overexpression of GAS6-AS1. D-E. Edu assays were performed to in A549 and PC9 cells after overexpression of GAS6-AS1. F-G. Transwell and Matrigel assays were used to test the migration and invasion ability of LUAD cells after overexpression of GAS6AS1, respectively. H. Images of xenograft tumors derived from nude mice-bearing A549 cells of different 
group. I. Tumor volume was measured every weeks after injection. J. Tumor weight was measured after resection of xenograft tumors.

A

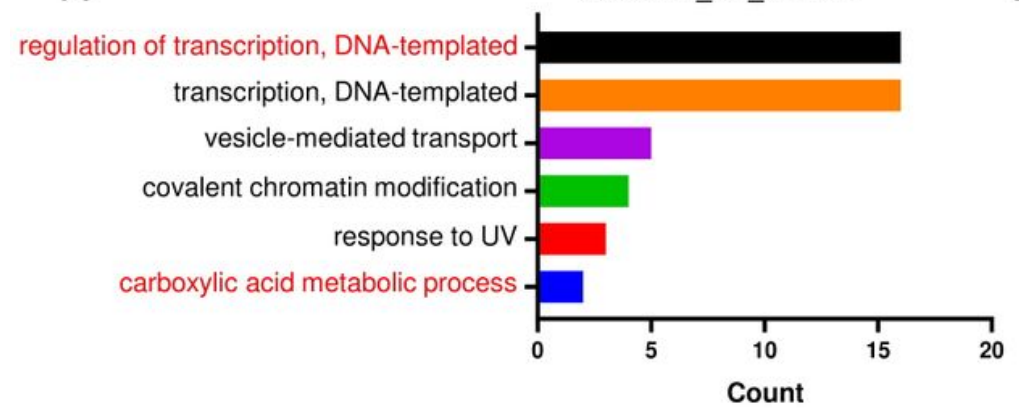

C

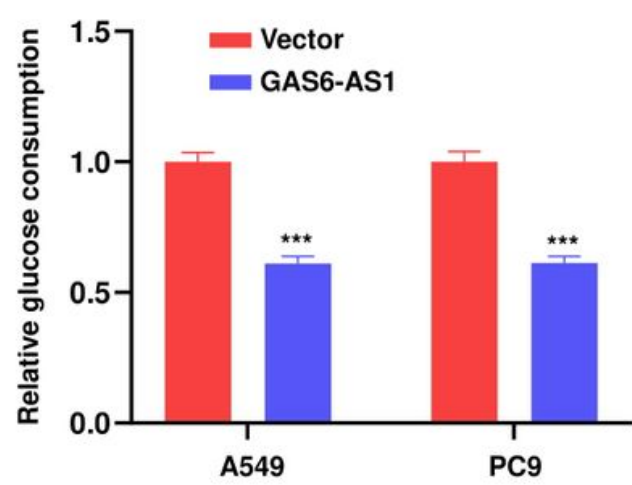

$\mathrm{F}$

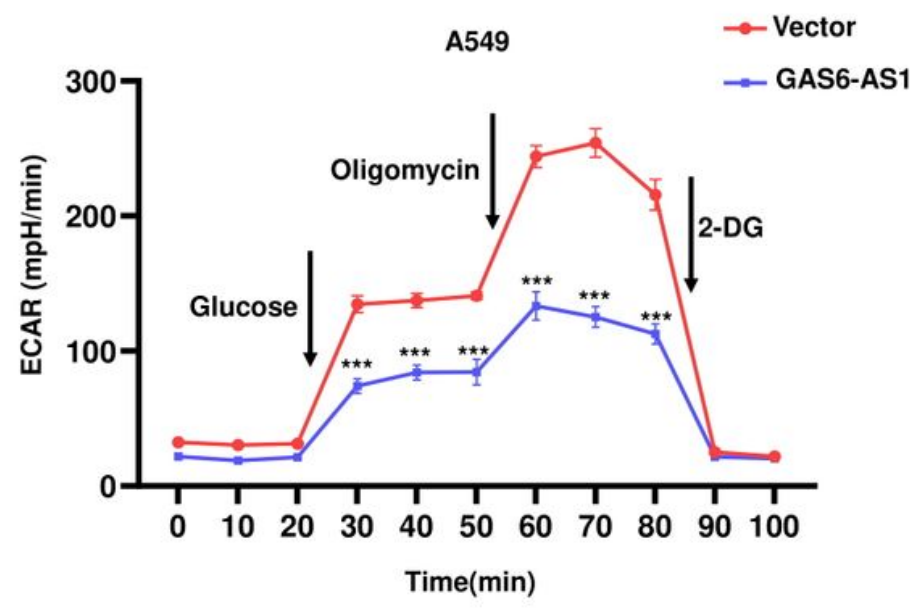

B

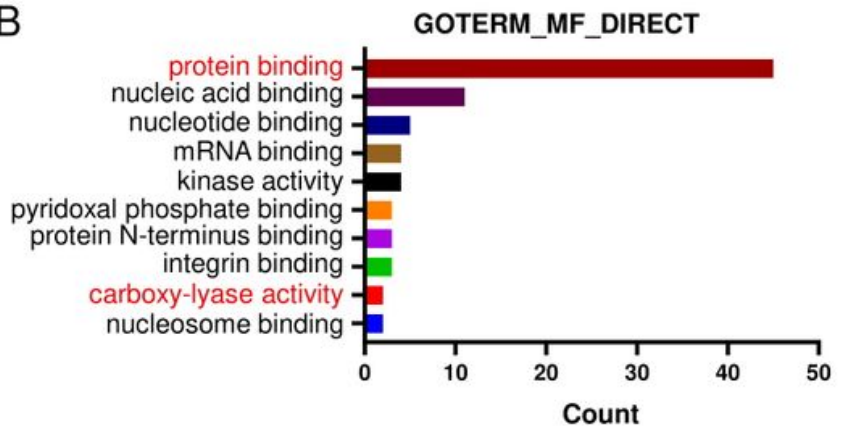

D

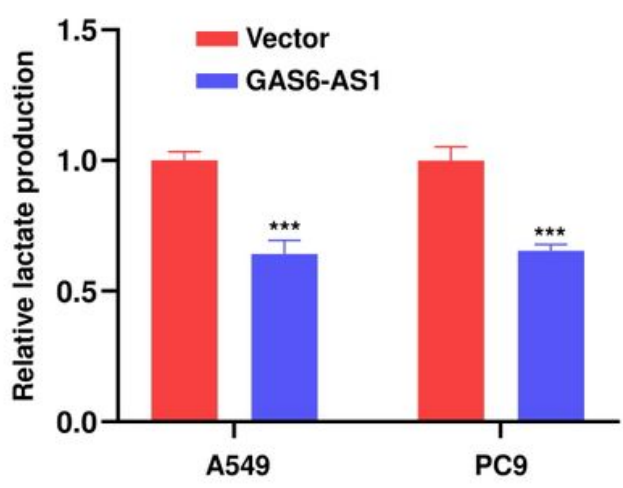

G
E

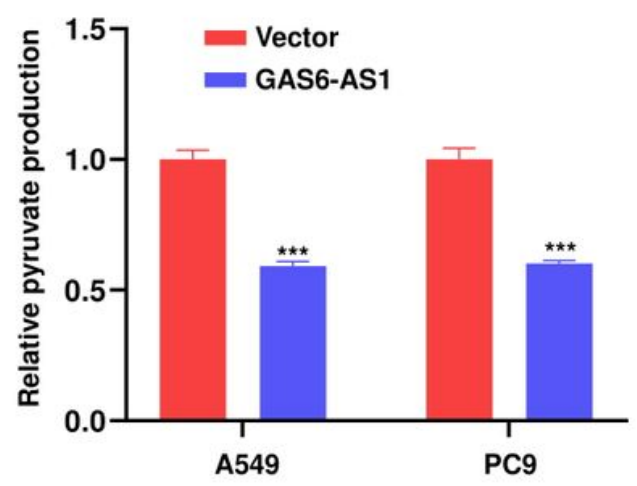

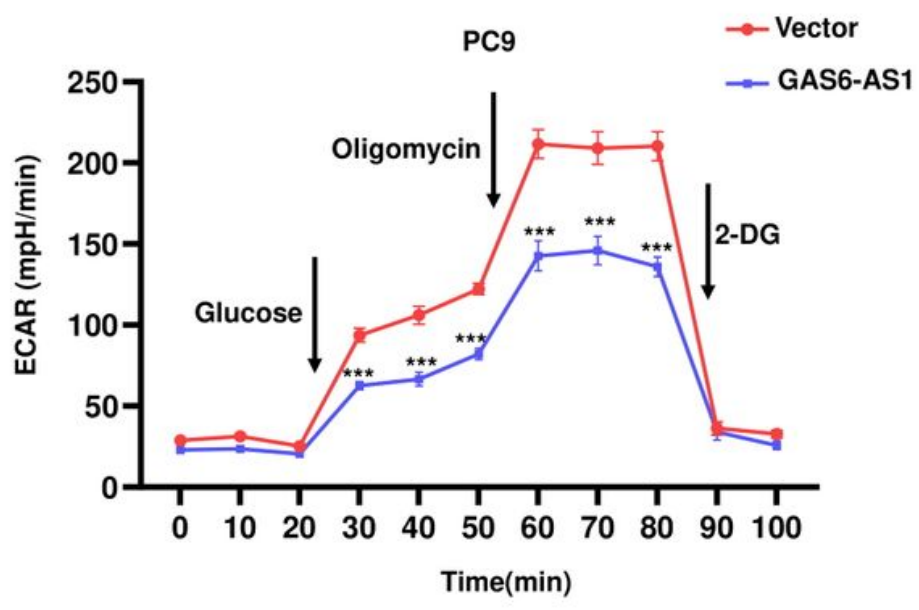

Figure 2

GAS6-AS1 suppressed glucose metabolism reprogramming in LUAD cells. A. Biological process of genes highly co-expressed with GAS6-AS1 by Gene_Ontology enrichment analysis. B. Molecular function of genes highly co-expressed with GAS6-AS1 by Gene_Ontology enrichment analysis. C. Detection of glucose consumption in LUAD cells after overexpression of GAS6-AS1. D-E. Detection of lactate and pyruvate production in LUAD cells after overexpression of GAS6-AS1. F-G. Extracellular acid ratio (ECAR) upon cells were measured by Seahorse XF in LUAD cells after overexpression of GAS6-AS1. 


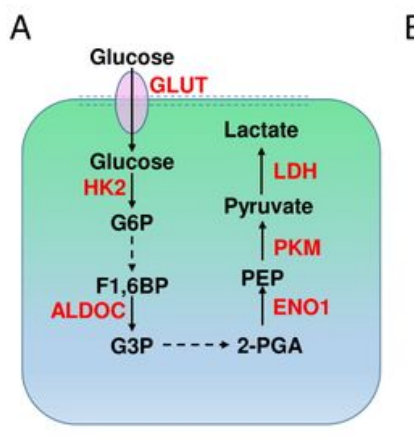

B

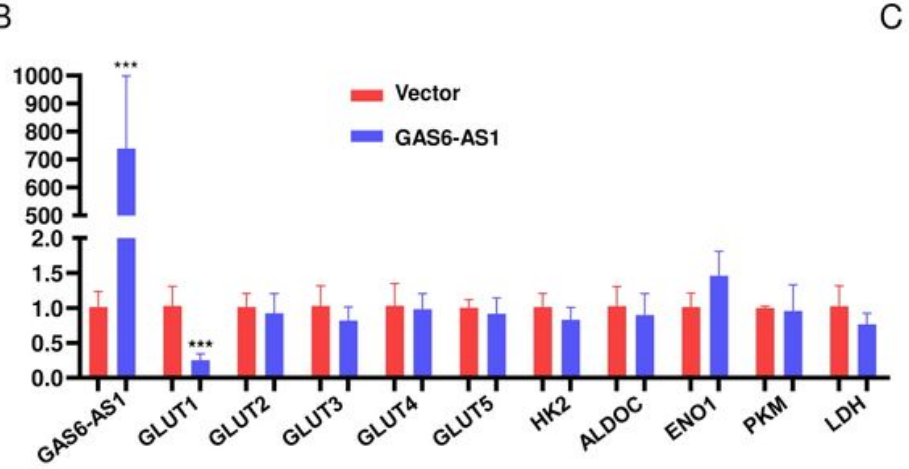

D

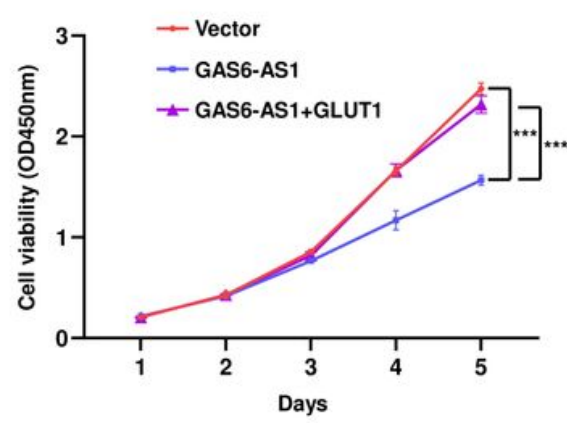

$\mathrm{E}$

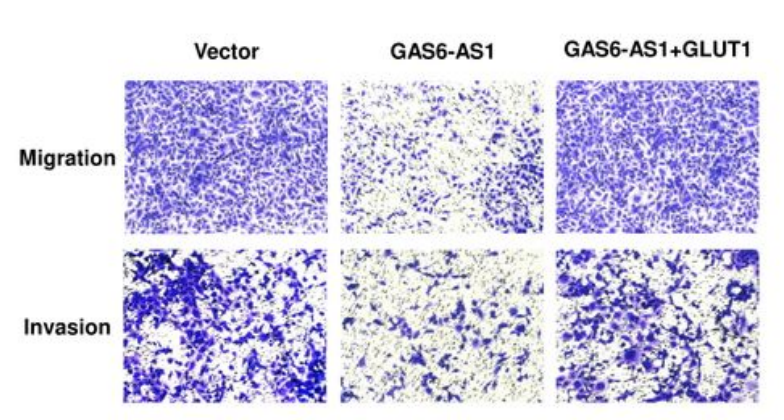

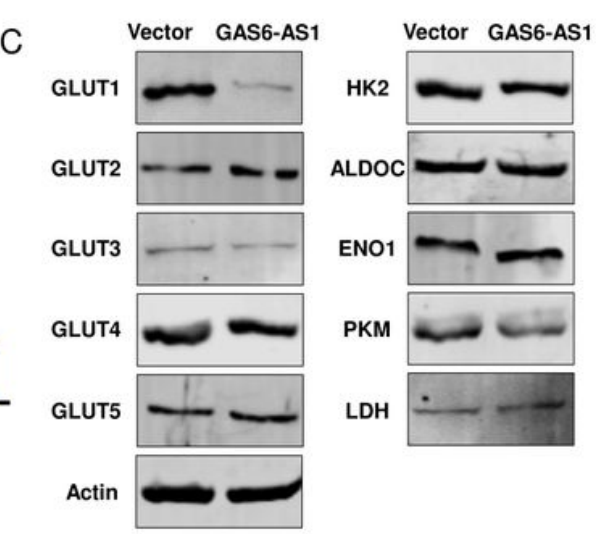

$\mathrm{F}$
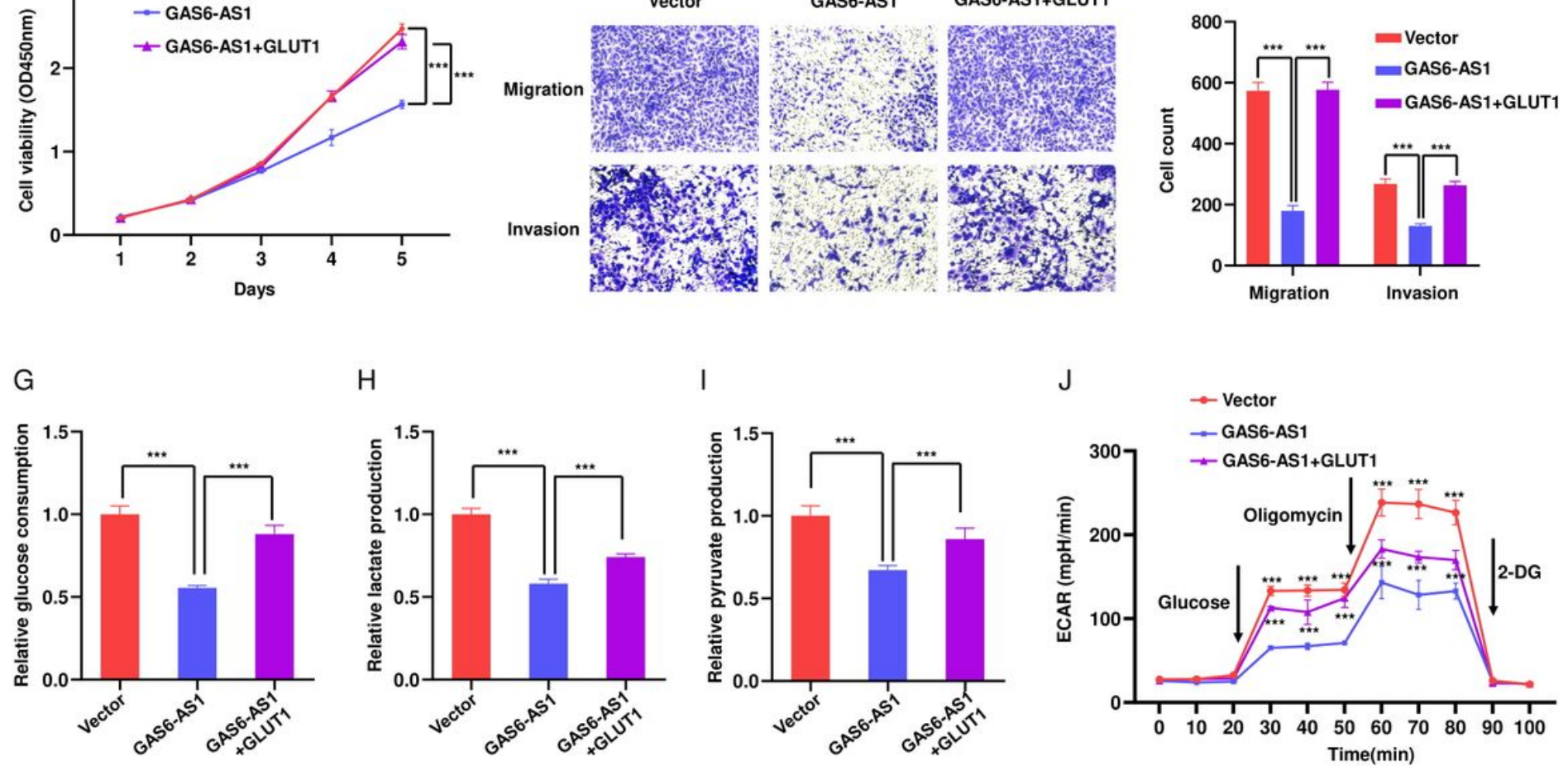

Figure 3

GAS6-AS1 inhibited progression and glucose metabolism reprogramming of LUAD cells via downregulating GLUT1. A. Diagram of key enzymes that play vital roles in glucose metabolism reprogramming. B. The mRNA expression of metabolism-related enzymes after overexpression of GAS6AS1 in A549 cells. C. The protein expression of metabolism-related enzymes after overexpression of GAS6-AS1 in A549 cells. D. Ectopic expression of GLUT1 restored the inhibition effect of GAS6-AS1 on proliferation in A549 cells. E-F. Ectopic expression of GLUT1 restored the inhibition effect of GAS6-AS1 on migration and invasion in A549 cells. G-J. Glucose consumption, lactate production, pyruvate production and extracellular acid ratio (ECAR) were measured, and results indicated that ectopic expression of GLUT1 restored the inhibition effect of GAS6-AS1 on glucose metabolism reprogramming in A549 cells. 

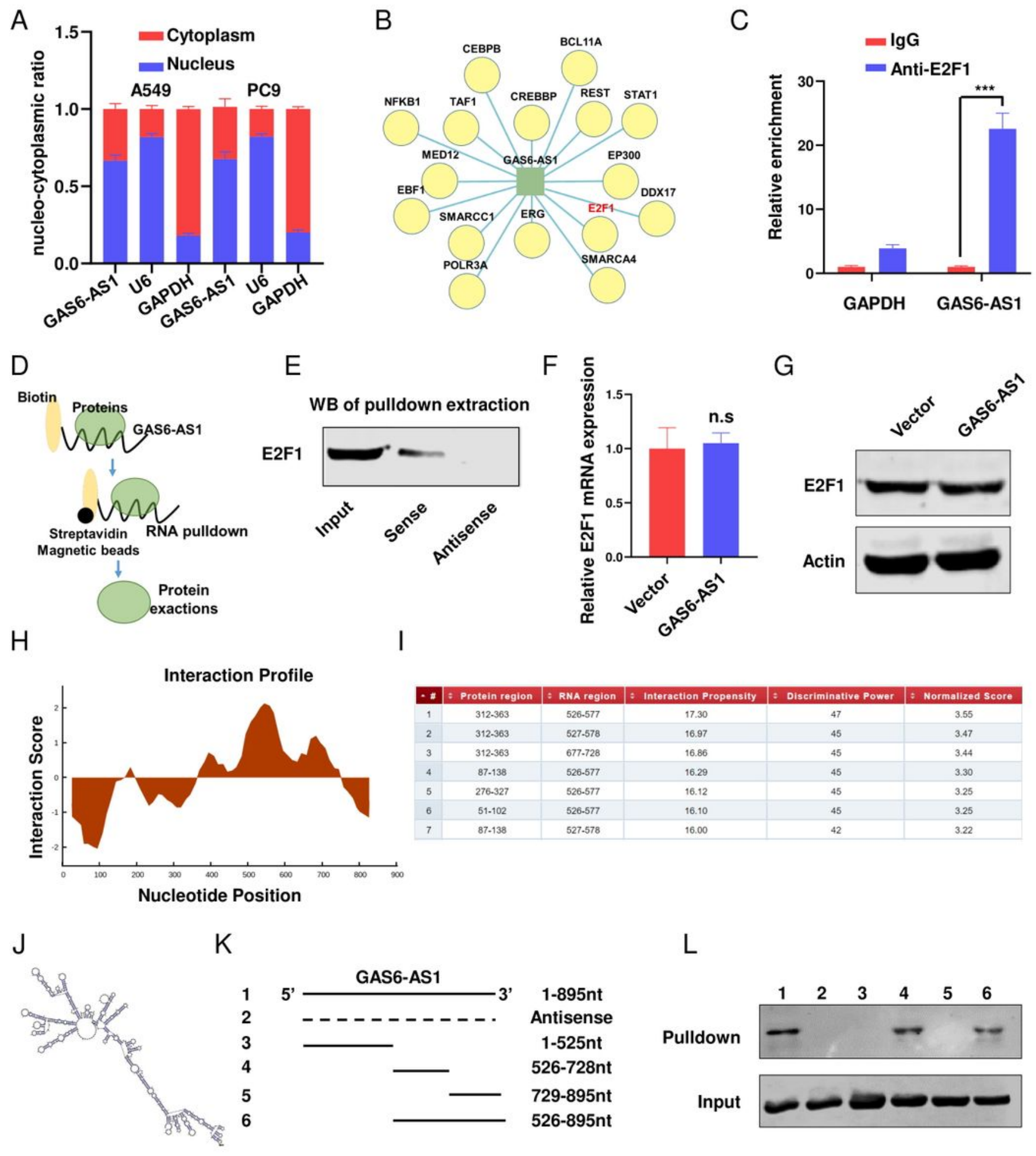

$\mathrm{L}$

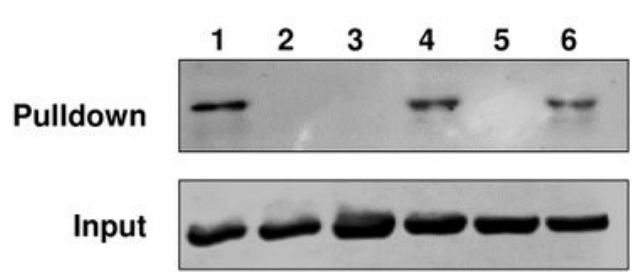

Figure 4

GAS6-AS1 interacted with transcription factor E2F1. A. RNA expression was measured after nuclear and cytosolic separation. U6 was used as a nucleus marker and GAPDH was used as a cytosol marker. B. Transcription factors that are predicted to interact with GAS6-AS1 by LncMap. C. RIP experiments were performed in A549 cells with E2F1 antibody and IgG was used as control. D. Schematic diagram of RNA pulldown assay. E. Lysates from A549 cells were used for RNA-pulldown with biotinylated GAS6-AS1 
transcript or antisense transcript. Western blotting demonstrated the specific association of GAS6-AS1 with E2F1. F-G. qRT-PCR and western blotting were performed to detect the mRNA and protein expression of E2F1 after overexpression of GAS6-AS1. H-I. The interaction profile and binding region of GAS6-AS1 with E2F1 predicted by catRAPID tools. J. The secondary structure of GAS6-AS1 in Annolnc. K. A series of GAS6-AS1 deletion mapping fragments were constructed and RNA pulldown assays were performed. L. Western blot analysis of the proteins retrieved from pulldown assays were performed and Input was used as positive control.

A
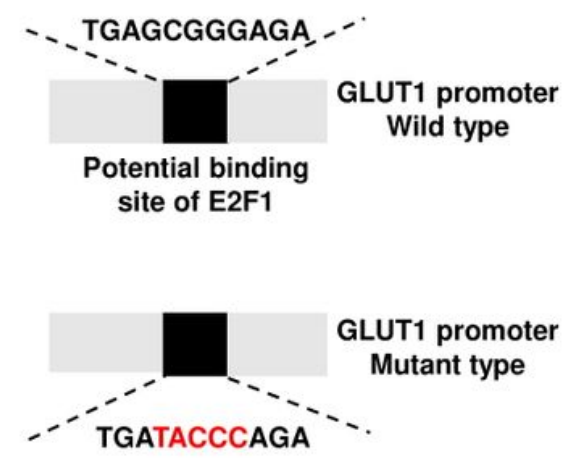

D

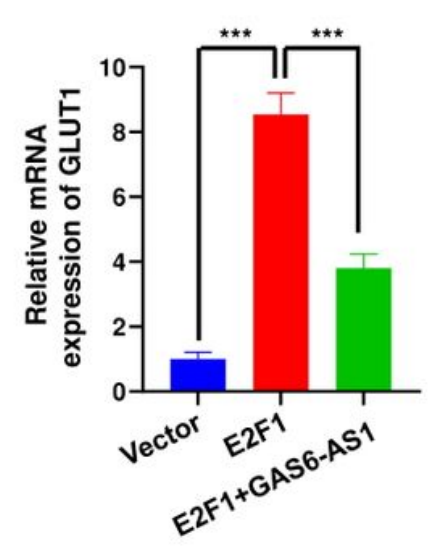

$E$
$\mathrm{B}$
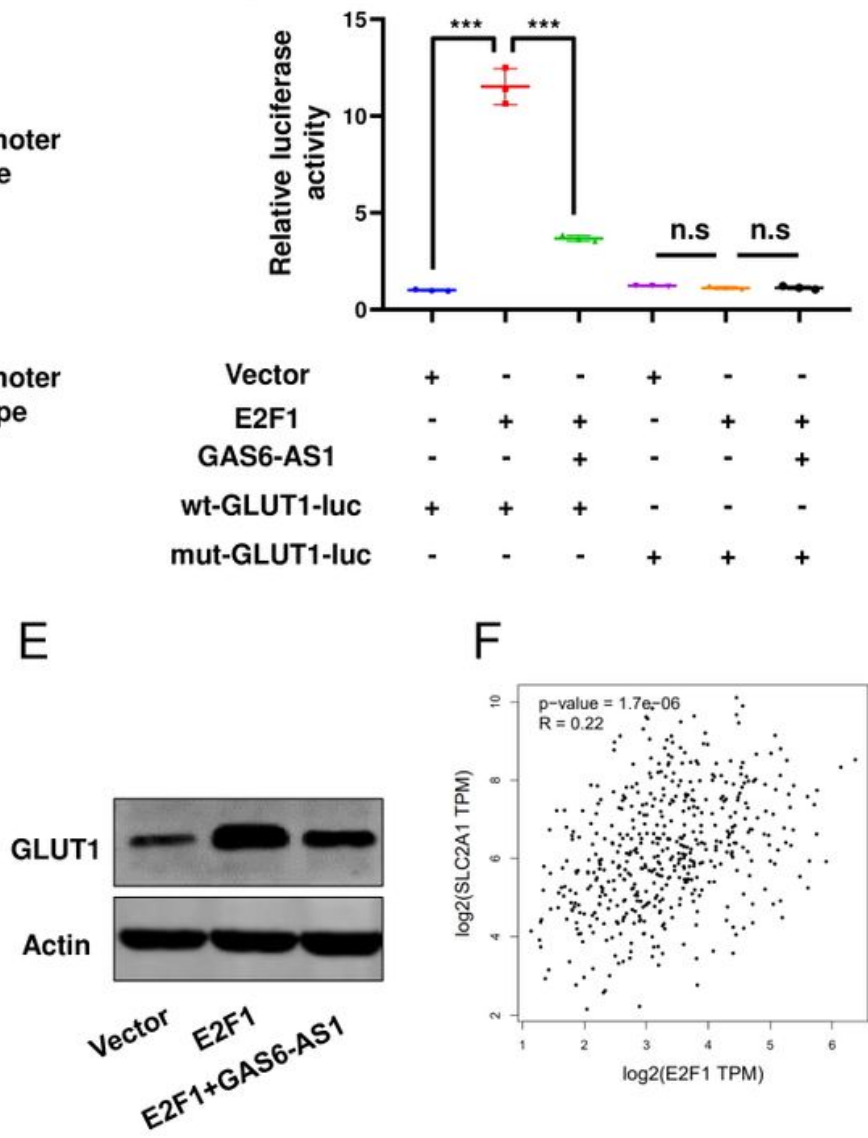

C
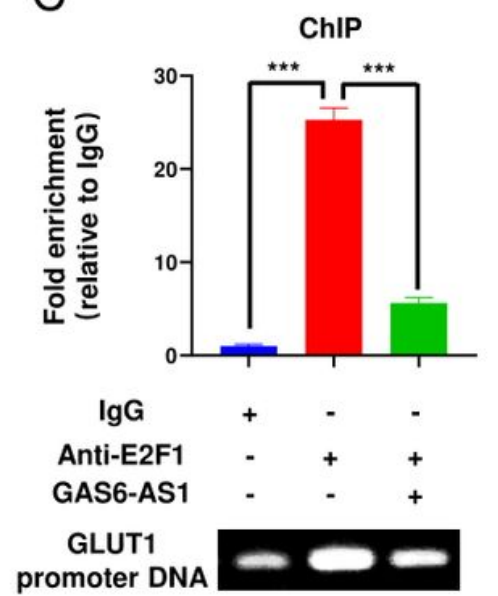

$\mathrm{G}$

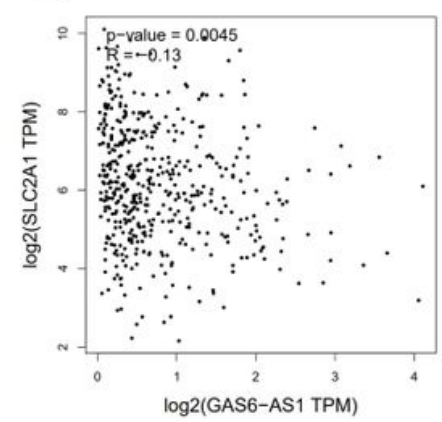

Figure 5

GLUT1 was a transcriptional target of E2F1, and the transcription was repressed by GAS6-AS1. A. The potential binding site of E2F1 with GLUT1 promoter was shown and the wild-type or mutant-type luciferase vectors were constructed. B. Luciferase activity was assayed in A549 cells transfected with luciferase vectors (wild type or mutant type) and meantime co-transfected with expression plasmids (empty vectors, E2F1 expression plasmids or GAS6-AS1 expression plasmids). C. ChIP experiments of E2F1 (IgG as control) was performed and the coprecipitated DNA was subjected to PCR amplification with primers specific to GLUT1 promoter region. D-E. The level of GLUT1 under ectopic expression of E2F1 or GAS6-AS1 was detected by qRT-PCR and western blotting. F-G. The correlation between E2F1 and GAS6-AS1 with GLUT1 in LUAD was presented. 
A

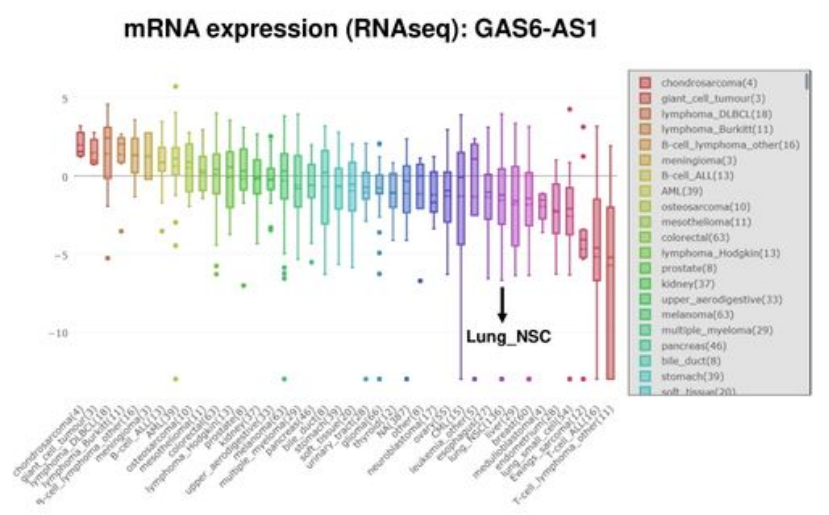

D

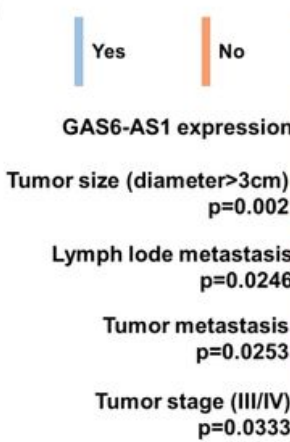

Low expression
B

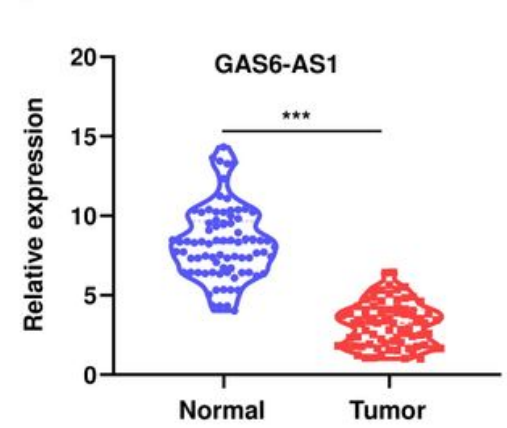

C

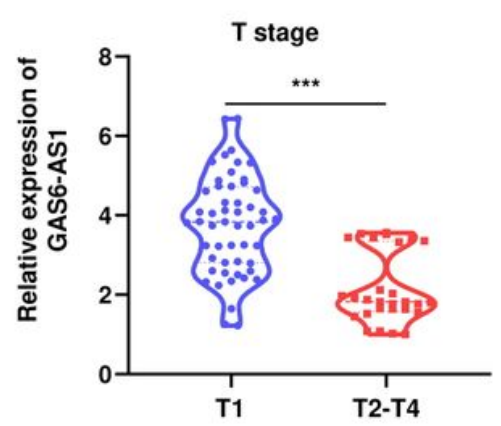

F

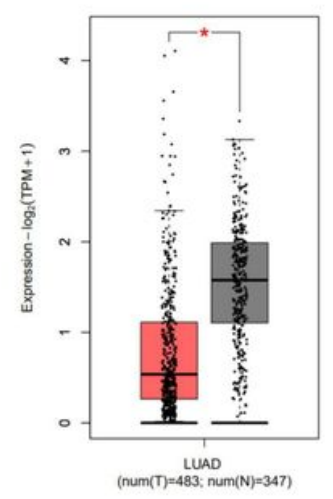

High expression

$\mathrm{H}$

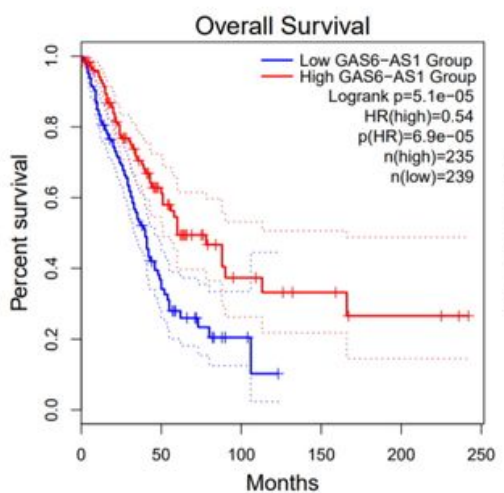

E

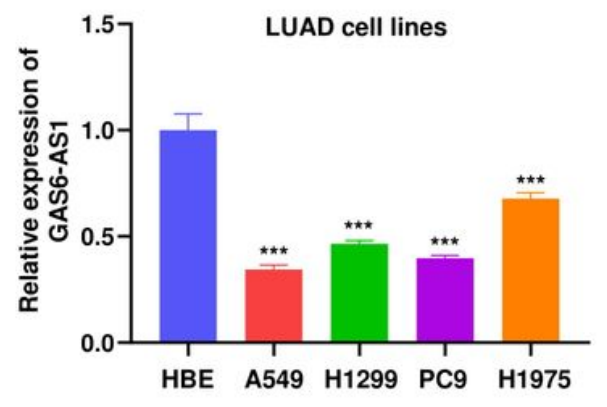

\section{Figure 6}

GAS6-AS1 was downregulated in LUAD tissues and correlated with clinical characteristics of LUAD patients. A. The expression of GAS6-AS1 in different cancer cells was shown from CCLE datasets. B. GAS6-AS1 was downregulated in 80 LUAD tissues compared with paired adjacent normal tissues. C. Patients of advanced T stages exerted lower expression of GAS6-AS1. D. Expression of GAS6-AS1 correlated with tumor size, lymph node metastasis, cancer distant metastasis and TNM stage of LUAD. E. Glucose starvation induced downregulation of GAS6-AS1 in A549 and PC9 cells. F. GAS6-AS1 was lowly expressed in LUAD tissues. G-I. The expression of GAS6-AS1 in LUAD correlated with tumor stage, overall survival and disease free survival of patients in GEPIA dataset. 


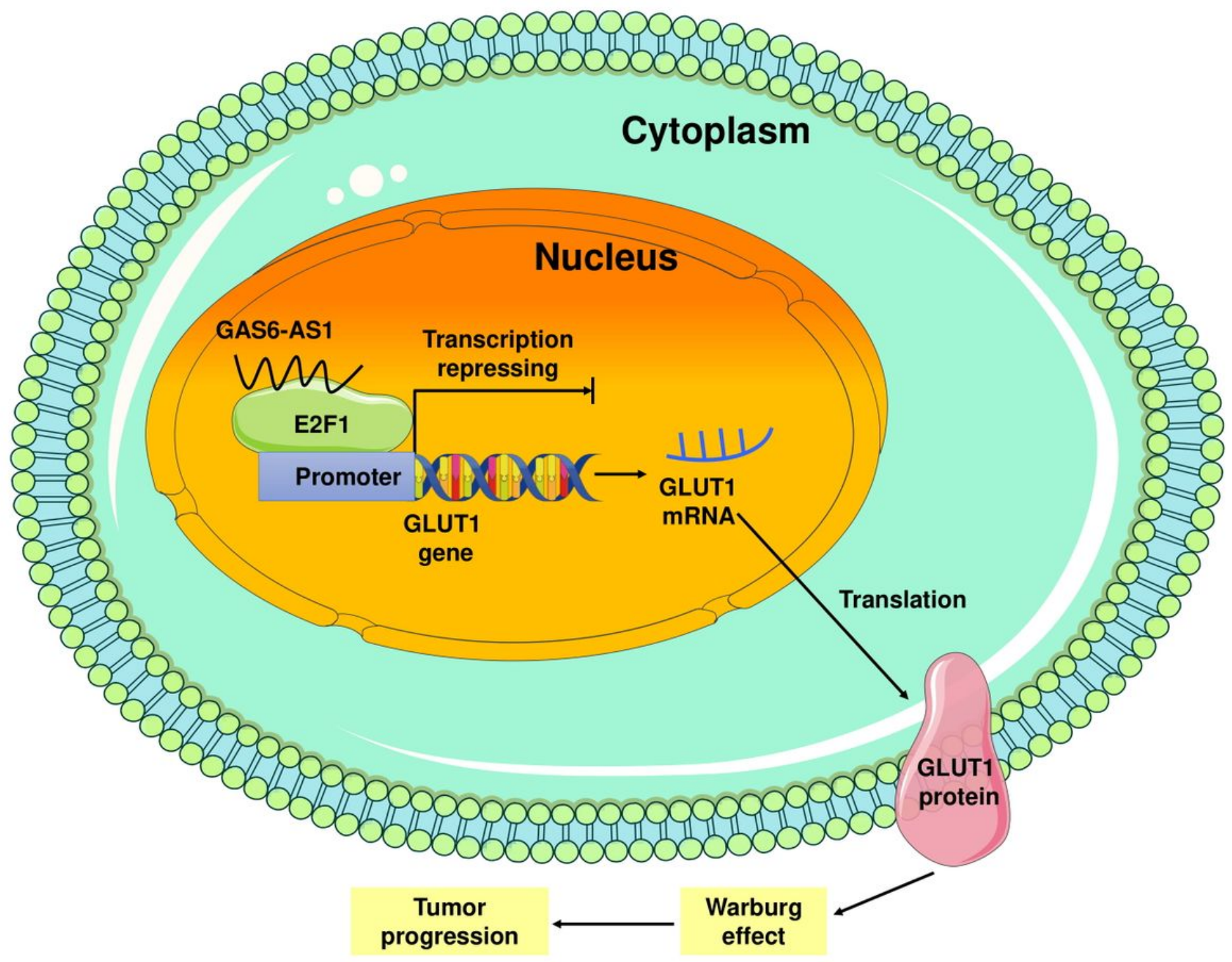

Figure 7

Schematic illustration of GAS6-AS1 in inhibiting glycolytic metabolism and progression of LUAD.

\section{Supplementary Files}

This is a list of supplementary files associated with this preprint. Click to download.

- FigureS2.pdf

- FigureS1.pdf

- TableS2.xIsx

- TableS1.xlsx 